\title{
The collateral network concept: Remodeling of the arterial collateral network after experimental segmental artery sacrifice
}

\author{
Christian D. Etz, MD, PhD, ${ }^{\mathrm{a}, \mathrm{c}}$ Fabian A. Kari, MD, ${ }^{\mathrm{a}, \mathrm{d}}$ Christoph S. Mueller, MD, ${ }^{\mathrm{a}}$ Robert M. Brenner, MS, ${ }^{\mathrm{a}}$ \\ Hung-Mo Lin, $\mathrm{PhD},{ }^{b}$ and Randall B. Griepp, $\mathrm{MD}^{\mathrm{a}}$
}

\begin{abstract}
Objective: A comprehensive strategy to prevent paraplegia after open surgical or endovascular repair of thoracoabdominal aortic aneurysms requires a thorough understanding of the response of the collateral network to extensive segmental artery sacrifice.
\end{abstract}

\begin{abstract}
Methods: Ten Yorkshire pigs underwent perfusion with a low-viscosity acrylic resin. With the use of cardiopulmonary bypass, 2 animals each were perfused in the native state and immediately, 6 hours, 24 hours, and 5 days after sacrifice of all segmental arteries (T4-L5). After digestion of surrounding tissue, the vascular cast of the collateral network underwent analysis of arterial and arteriolar diameters and the density and spatial orientation of the vasculature using light and scanning electron microscopy.
\end{abstract}

Results: Within 24 hours, the diameter of the anterior spinal artery had increased significantly, and within 5 days the anterior spinal artery and the epidural arterial network had enlarged in diameter by $80 \%$ to $100 \%(P<.0001)$. By 5 days, the density of the intramuscular paraspinous vessels had increased $(P<.0001)$, a shift of size distribution from small to larger arterioles was seen $(P=.0002)$, and a significant realignment of arterioles parallel to the spinal cord had occurred $(P=.0005)$.

Conclusions: Within 5 days after segmental artery occlusion, profound anatomic alterations in the intraspinal and paraspinous arteries and arterioles occurred, providing the anatomic substrate for preservation of spinal cord blood flow via collateral pathways. (J Thorac Cardiovasc Surg 2011;141:1029-36)

Recent clinical studies have suggested that a significant proportion of the increasingly uncommon, but still tragic, cases of paraplegia and paraparesis after thoracoabdominal aortic aneurysm (TAAA) repair are no longer apparent immediately, but occur postoperatively. ${ }^{1-3}$ The probability of delayed spinal cord injury seems to decrease as the interval after operation lengthens, suggesting a time-dependent adaptation to the loss of segmental artery (SA) input sacrificed at surgery or, inevitably, during endovascular repair. ${ }^{2}$

Previous investigations have shown that an estimate of spinal cord perfusion pressure (the pressure measured in the distal end of a ligated intercostal or lumbar vessel) decreases substantially with extensive SA sacrifice, but recovers toward pre-segmental vessel ligation values within a few days. ${ }^{4}$ In the accompanying publication, ${ }^{5}$ the existence of an extensive collateral network of arteries that link the

\footnotetext{
From the Department of Cardiothoracic Surgery, ${ }^{a}$ Department of Anesthesiology/Division of Biostatistics, ${ }^{\mathrm{b}}$ Mount Sinai School of Medicine, New York, NY; Department of Cardiac Surgery, ${ }^{\mathrm{c}}$ Heartcenter, Leipzig University, Leipzig, Germany; and Department of Cardiovascular Surgery, Cardiovascular Center, University Medical Center Freiburg, ${ }^{\mathrm{d}}$ Freiburg, Germany.

Funding: grant HL0456636 from the National Heart, Lung, and Blood Institute.

Disclosures: Authors have nothing to disclose with regard to commercial support.

Received for publication April 13, 2010; revisions received May 25, 2010; accepted

for publication June 8, 2010 .

Address for reprints: Randall B. Griepp, MD, Department of Cardiothoracic Surgery,

Mount Sinai School of Medicine, One Gustave L. Levy Place, New York, NY

10029 (E-mail: randall.griepp@mountsinai.org).

$0022-5223 / \$ 36.00$

Copyright (C) 2011 by The American Association for Thoracic Surgery

doi:10.1016/j.jtcvs.2010.06.017
}

circulation of the spinal cord, the paravertebral tissues, and the skeletal muscles of the back both axially and transversely is described. Our hypothesis is that the anatomic changes that occur after the sacrifice of the SA input into this network provide a robust blood supply based on input from extrasegmental sources within a few days. The current study demonstrates those anatomic changes, which allow the recovery of spinal cord perfusion pressure in the first few days after complete SA sacrifice, using acrylic injection casts ${ }^{6,7}$ of the relevant vasculature in the juvenile pig.

\section{MATERIALS AND METHODS}

Ten female juvenile Yorkshire pigs (Animal Biotech Industries, Allentown, $\mathrm{NJ}$ ) weighing $12 \pm 2$ (range, 10-13) kg underwent standard aortic cannulation and total body perfusion with a low-viscosity acrylic resin $(800 \mathrm{~mL}$, Batsons Nr 17, Anatomical Corrosion Kit, Polysciences Inc, Warrington, PA, www.polysciences.com) to create a vascular cast of the circulation in the native state and immediately, 6 hours, 24 hours, and 5 days after SA sacrifice (T4-L5). Perfusion was carried out using extracorporeal circulation, as described in detail in the accompanying publication. ${ }^{5}$

After curing of the resin and digestion of all organic tissue as previously described, the anatomy of the blood supply to the spinal cord, and especially its interconnections with the vasculature of adjacent muscles, was studied grossly and in detail using light and electron microscopy. The vessels contributing to the blood supply of the spinal cord were visualized, and their characteristics were evaluated.

\section{Perioperative Management, Anesthesia, Acrylic Perfusion, and Cast Processing}

All animals received humane care in compliance with the guidelines of Principles of Laboratory Animal Care, formulated by the National Society 


\author{
Abbreviations and Acronyms \\ ASA = anterior spinal artery \\ SA $=$ segmental artery \\ SEM = scanning electron microscopy \\ TAAA $=$ thoracoabdominal aortic aneurysm
}

for Medical Research, and the Guide for the Care and Use of Laboratory Animals, published by the National Institutes of Health (Publication No. 88-23, revised 1996). The Mount Sinai Institutional Animal Care and Use Committee approved the protocols for all experiments.

Anesthetic management, acrylic resin perfusion, cast processing, and analysis by light and scanning electron microscopy (SEM) were carried out as described in detail in the accompanying publication. ${ }^{5}$

\section{Segmental Artery Sacrifice}

Eight of the 10 pigs underwent complete serial SA sacrifice (T4-L5). In all 8 animals, the descending thoracic and abdominal aorta were exposed, and all SAs $\left(\mathrm{T}_{4-13}, \mathrm{~L}_{1-4 / 5}\right)$ were carefully dissected. Thereafter, all thoracic and abdominal SAs were sequentially clamped in a craniocaudal direction during mild hypothermia $\left(32^{\circ} \mathrm{C}\right)$, allowing a 3-minute interval between clamping of successive arteries.

Four pigs remained under anesthesia for acrylic resin infusion, which was achieved immediately after SA sacrifice in 2 animals and 6 hours after SA sacrifice in 2 animals. Four pigs were allowed to recover from anesthesia and underwent acrylic resin perfusion 1 day $(\mathrm{N}=2)$ or 5 days $(\mathrm{N}=2)$ after SA sacrifice. Two pigs underwent acrylic resin perfusion without previous SA sacrifice to serve as controls; they were sacrificed immediately. No delayed sacrifice in controls was carried out.

\section{Cast Analysis}

The anatomy of the spinal cord blood supply and its connections with the vasculature of adjacent muscles were investigated macroscopically and microscopically (light and SEM). Each cast was analyzed with a focus on the axial arterial network in the spinal canal and paraspinous muscles. Collateral anastomoses were identified, and the vessel network was described in detail, quantifying vessel density and diameters.

\section{Light Microscopy}

After opening the spinal canal by removing the dorsal processes and washing the intraspinal portion of the cast, images of the intraspinal vessels (anterior spinal artery [ASA] and epidural arcades) at every segmental level were obtained. The digital image files were used for measurements of intraspinal vessel diameters using ImageTool 3.0 image analysis software (University of Texas Health Science Center at San Antonio).

\section{Scanning Electron Microscopy}

A scanning electron microscope (Hitachi S 4300 scanning electron microscope; Hitachi High-Tec, Roslyn Heights, NY; cold field SEM, http:/ www.hitachi-hitec.com/global/em/fe/fe_index.html) was used to visualize the microstructure of the arterial network. Each sample was cleaned in a warm distilled water bath to remove organic remnants and calcium for a minimum of 10 days before gold coating for SEM using a Technics Hummer V Sputter Coater (Stanford Nanofabrication Facility, Stanford, Calif).

SEM imaging data were systematically acquired. Angio computed tomography scanning and 3-dimensional reconstructions of selected casts allowed for digital processing and collection of data regarding spatial configuration. The studies were designed to enable accurate quantification of size, density, and orientation of the vascular network supplying both the spinal cord and the muscles and other tissues adjacent to it.

\section{Scanning Electron Microscopy Quantification of Vessel Diameter Distribution}

For morphometric analysis of the vascular networks, an overview image $(\times 80)$ was used to identify representative areas of the vascular cast specimen with good perfusion. Pictures were taken of these areas at various magnifications $(\times 100, \times 200, \times 400)$. All image data were stored as digital files and processed for morphometric analysis. Contrast within each image file was adjusted to achieve optimal visualization of as many vessels as possible. A counting grid (35 single squares, $0.034 \mathrm{~mm}^{2}$ each, images $\times 100$ ) was projected onto the original image file with an $\mathrm{x}$ - and $\mathrm{y}$-axis in every square (Adobe Photoshop CS 2; Adobe Systems Inc, San Jose, Calif). The grid was used for spatial orientation within the image and to ensure an even and random distribution of measurements. The vessels to be counted and measured were chosen from lists of random numbers used as coordinates within the counting grid. Two measurements per square were performed using ImageTool 3.0 image analysis software.

The density of the vasculature was determined by calculating the frequency with which vessels were seen within the area selected for morphometric analysis, as described earlier. Arterial vessels were identified according to SEM morphologic criteria defining arterioles: sharply demarcated and longitudinally oriented endothelial nuclear imprints, oval in shape. ${ }^{8}$ Size distribution of arterioles was determined by measuring the vessels encountered during morphometric analysis.

For a sense of the dynamic process of adaptation of the collateral system, values from the 2 pigs without SA sacrifice-also labeled native-were pooled to provide baseline measurements. These results were then compared with average measurements from the pigs sacrificed immediately, at 6 hours, 24 hours, and 5 days after serial sacrifice of all SAs. The casts from the pigs at each time point were compared with the native casts to establish a general framework of the changes that occur after SA sacrifice and to ascertain which observations should be quantified. The major effort involved in quantification was restricted to analysis of the results at 24 hours and 5 days. Earlier casts showed uneven filling of the mid-portion of the cord and what remained was so fragile that its accuracy was thought to be suspect. Some later casts— those at 24 hours after sacrifice—-showed no dramatic changes in some regions compared with native casts, and therefore quantification was not systematically undertaken. The text describes the changes as though they were serial measurements in the same animal over time to make it easier to visualize the process of vascular remodeling. Pilot studies in native animals had demonstrated little size variation in similar vessels in animals of the same weight. ${ }^{5}$

\section{Statistical Methods}

Data were entered in an Excel spreadsheet (Microsoft Corp, Redmond, WA) and transferred to an SAS file (SAS Institute Inc, Cary, NC) for data description and analysis; data are described as percents, median (range), or means (standard deviation).

\section{RESULTS}

The vascular system supplying the spinal cord has extensively interconnected intraspinal and paraspinal components. The intraspinal system, fed by the radiculomedullary branches of the SAs, feeds the anterior spinal artery (ASA), which has multiple connections with a longitudinally continuous system of epidural artery arcades. The adjacent paraspinous system is a quantitatively larger vascular network supplying the vertebrae and the erector spinae and psoas muscles (Figure 1). This entire vascular network was 


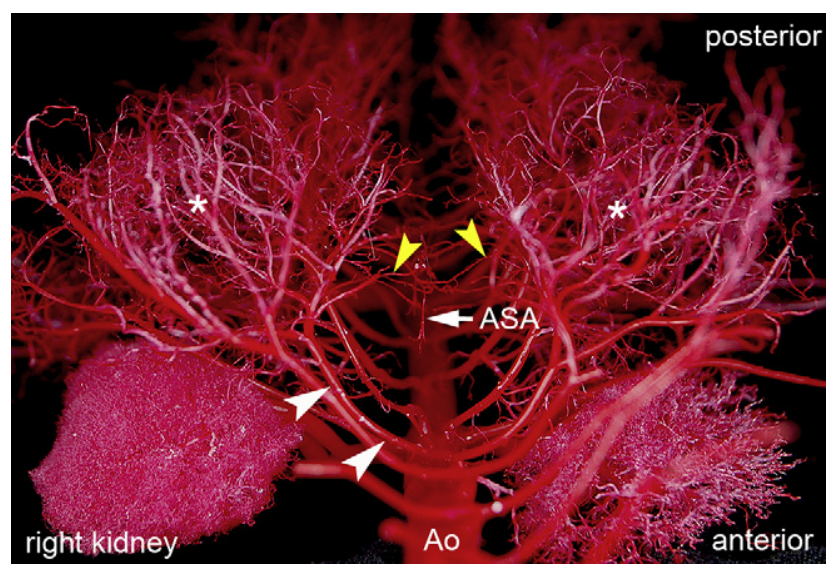

FIGURE 1. Vascular cast of the abdominal aorta, renal arteries, kidneys, and dorsal lumbar segmental arteries (SAs) in a Yorkshire pig (cranio-caudal view, segment L1 in foreground). Soft tissue and some bony structures have been completely removed to demonstrate the distribution and branching of dorsal SAs. White arrowheads show the main trunks of the SAs following the contour of the removed vertebral bodies. Note the extensive contribution of the SAs to the paraspinous musculature (asterisks). Two to 3 branches of each SA trunk reach the intraspinal collateral network (yellow arrowheads) and the ASA. ASA, Anterior spinal artery; Ao, abdominal aorta.

cast in its entirety in each animal. The polymeric resin reached networks of small arterioles, capillaries (with diameters $<7 \mu \mathrm{m}$ ), and venules. In the studies done less than 24 hours after SA sacrifice, the filling of the vasculature in the mid-portion of the cord was uneven and the cast was fragile, allowing us to form an impression of the changes, but precluding reliable quantitative analysis.

Previous cast studies had identified the general outlines of the paraspinous collateral system ${ }^{7}$ and prompted its quantitative analysis in terms of the density of vessels, the distribution of capillaries and of arterioles of various diameters, and their orientation with respect to the spinal cord. ${ }^{5}$ The earlier cast studies also defined the intraspinal vascular supply of the cord as consisting principally of the ASA and a previously undescribed extensive series of longitudinally connected hexagons comprising an epidural network that has multiple connections with the ASA and extends the length of the cord. ${ }^{5}$ Because previous clinical and experimental evidence suggested that the impact of SA sacrifice in different regions of the cord might be different, the analysis for both intraspinal and paraspinal vascular beds was carried out separately for the upper thoracic, lower thoracic, and lumbar regions.

\section{Changes in the Diameter of the Anterior Spinal Artery}

The upper thoracic ASA diameter increased from 142 to $243 \mu \mathrm{m}$ at 5 days (corresponding to a change in crosssectional area from 0.016 to $0.044 \mathrm{~mm}^{2}$ ) and from 134 to

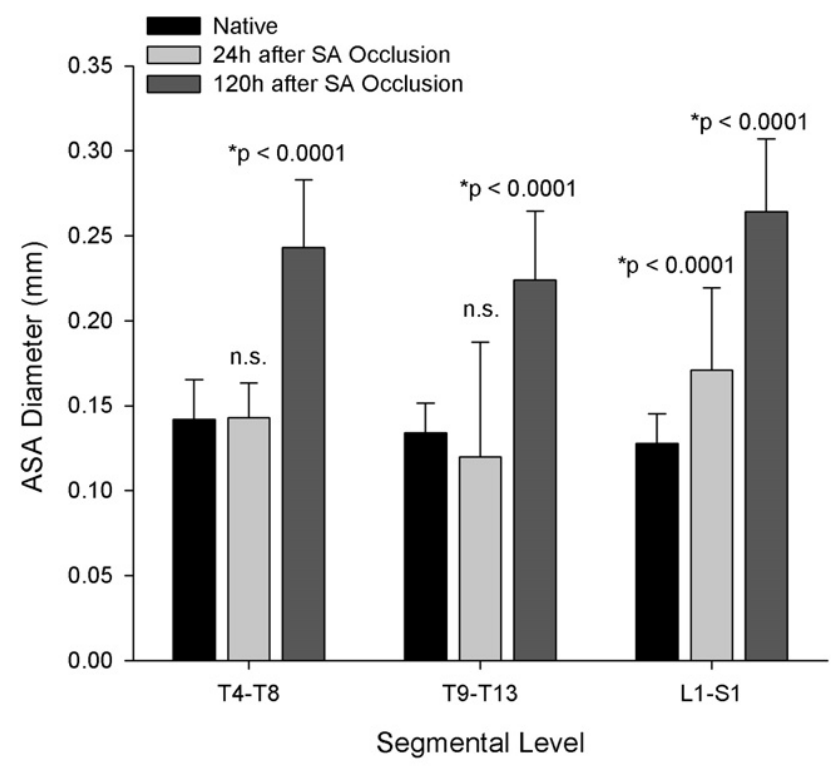

* all p-values count for Least Squares Means compared to Native

FIGURE 2. Increase in the diameter of the ASA 24 hours and 5 days after occlusion of all segmental arteries in the pig model. Native: patent segmental inflow into collateral networks. Separate measurements of the upper thoracic, lower thoracic, and lumbar segments have been merged. ASA, Anterior spinal artery; $S A$, segmental artery.

$224 \mu \mathrm{m}$ in the lower thorax (cross-sectional area 0.014 vs $\left.0.039 \mathrm{~mm}^{2}\right)(P<.0001)$. The lumbar ASA diameter also increased significantly, expanding from 127 to $171 \mu \mathrm{m}$ at 24 hours (cross-sectional area 0.013 vs $0.023 \mathrm{~mm}^{2}$ ) and to $264 \mu \mathrm{m}$ at 5 days (area $\left.0.055 \mathrm{~mm}^{2}\right)(P<.0001)$. Further details can be seen in Figure 2.

\section{Changes in the Epidural Vessels}

The epidural arcades, which form a chain surrounding the spinal cord that looks like a longitudinally connected series of hexagons, communicate with the ASA. As with the ASA, the diameter of the epidural arteries also increases dramatically in size within 5 days after SA sacrifice. Epidural arteries increased in diameter from 150 to $249 \mu \mathrm{m}$ at 5 days (cross-sectional area 0.018 vs $\left.0.049 \mathrm{~mm}^{2}\right)(P<.001)$, with greater enlargement below L1. Figure 3 depicts the quantitative analysis of diameter change from the native configuration to 5 days after SA ligation.

\section{Overview of Intraspinal Network Changes}

A light micrograph of the intraspinal components of the collateral network in the native pigs compared with the picture 5 days after SA sacrifice can be seen in Figure 4, complementing the account of their morphometric changes. This figure illustrates the striking enlargement of the components of the intraspinal collateral arterial network surrounding the spinal cord after the dramatic changes in input brought about by SA ligation. 


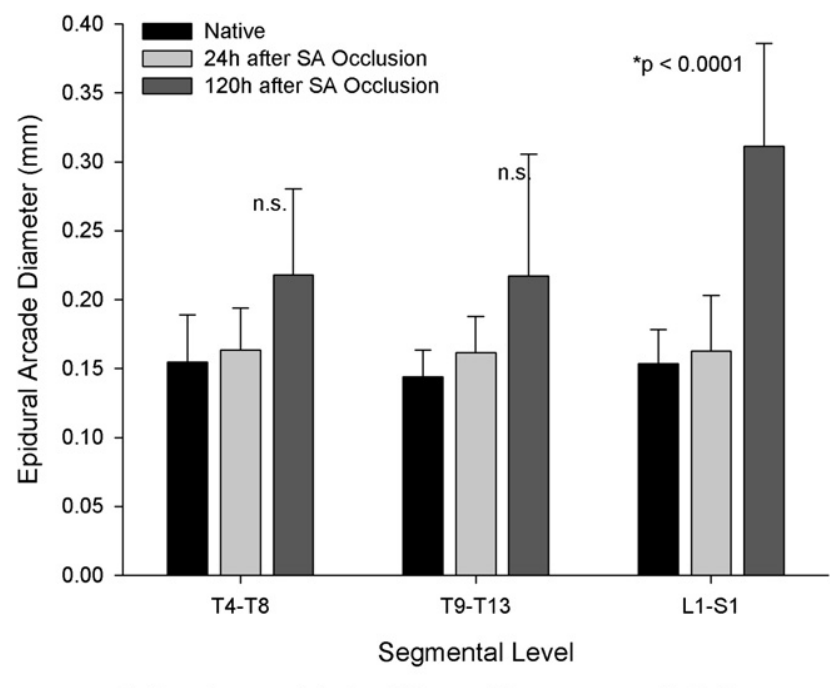

* all p-values count for Least Squares Means compared to Native

FIGURE 3. Epidural arcade diameter changes at 24 and 120 hours after complete SA ligation in the pig model. SA, Segmental artery; n.s., not significant.

\section{Density of Vessels Within the Intramuscular Collateral Network}

The density of the vasculature 5 days after SA sacrifice was compared with the density at baseline by determining how often randomly designated coordinates within each region included vessels. The density of the paraspinous vas- cular network as a whole was significantly increased within 5 days in the lower thoracic region $(P<.0001)$ (Figure 5).

\section{Remodeling of the Paraspinous Collateral Network}

Figure 6 demonstrates the dramatic shift in distribution of intramuscular vessel diameters in the lower thoracic and lumbar regions from capillaries and small arterioles to larger arterioles.

Progressive arteriolar realignment parallel to the spinal cord was also observed after extensive SA ligation. Figure 7 shows representative shifts in arteriolar alignment at the level of the lumbar cord from 40 degrees in the native state to 30 degrees at 24 hours, and to 9 degrees at 120 hours (5 days). This shift in alignment from the native state to 120 hours after SA ligation is quantified in Figure 8. Although a greater degree of parallelism is present in the lower thoracic and lumbar segments, realignment is seen throughout the paraspinous network 120 hours after SA ligation.

\section{Overview of Changes}

Profound changes occur in both the intraspinal and paraspinous vessels within 5 days of complete sacrifice of SAs. The ASA and the epidural arcades increase in diameter from $80 \%$ to $100 \%$. Cross-sectional areas increase 3- to 4-fold, with calculated flow resistance decreasing to $2 \%$ to $6 \%$ of baseline. In the paraspinous network, the vessel density increases throughout, with significant changes of $300 \%$ in the lower thorax. There is a substantial shift in vessel diameter

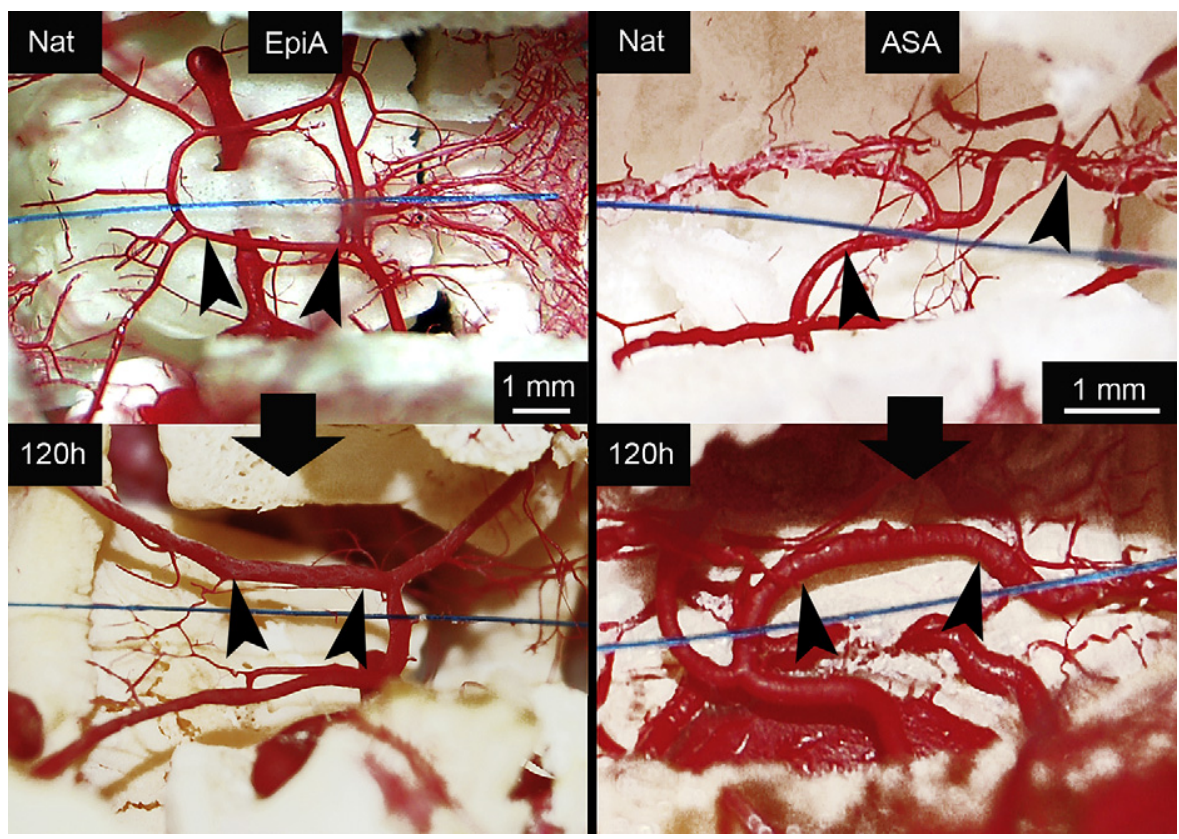

FIGURE 4. Increase in diameters of epidural arcades (left, black arrowheads) and ASA (right, black arrowheads) in the native pig compared with a pig perfused 5 days after extensive SA occlusion (120 hours). Soft tissue was removed completely from the samples, but the bony vertebral column was preserved. Note the specific enlargement of the longitudinally oriented vessels of the epidural arcade on the left. The blue line is a segment of prolene suture to impart and additional sense of scale. Nat, Native; EpiA, epidural arcade; ASA, anterior spinal artery. 


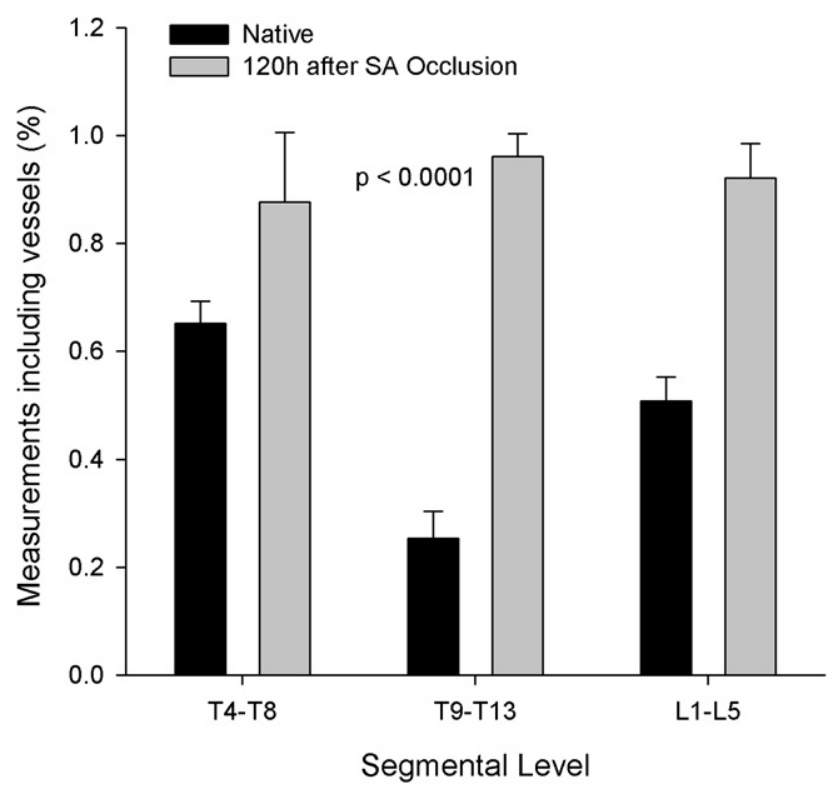

FIGURE 5. Paraspinous vessel density change 120 hours after complete SA ligation in the pig. Bars represent the percentage of randomly determined coordinates that included a blood vessel. SA, Segmental artery.

distribution toward larger vessels at the lower thoracic and lumbar levels. Finally, there is a highly significant realignment of paraspinous vessels from a random distribution to a parallel orientation along the axis of the spinal cord. Taken together, these provide a dynamic anatomical explanation of how the spinal cord survives when deprived of its segmental input.

\section{DISCUSSION}

This study of serial changes in the spinal cord blood supply during the first 5 days after SA sacrifice fills in significant details regarding the recovery of the collateral network. The time course of recovery of perfusion had been pieced together from previous experimental and clinical studies that included monitoring of blood flow and pressure. ${ }^{4,9,10}$ The current study gives us a more complete picture of the anatomic mechanisms that explain how the collateral network responds to its loss of segmental inputs during the first few days postoperatively.

There is dilatation within 24 hours of various vessels involved in the recovery of spinal cord perfusion pressure, but it takes approximately 5 days for structural remodeling of the intraspinal arteries and the collateral system. Increases in the density and mean diameters of arteries and arterioles are apparent both in the epidural intraspinal network and in the paraspinous muscular component, especially at the caudal and cranial ends of the simulated aortic resection. There is also a realignment of the paraspinous vessels parallel to the spinal cord, but it is not apparent what role this realignment plays in spinal cord blood flow. The realignment
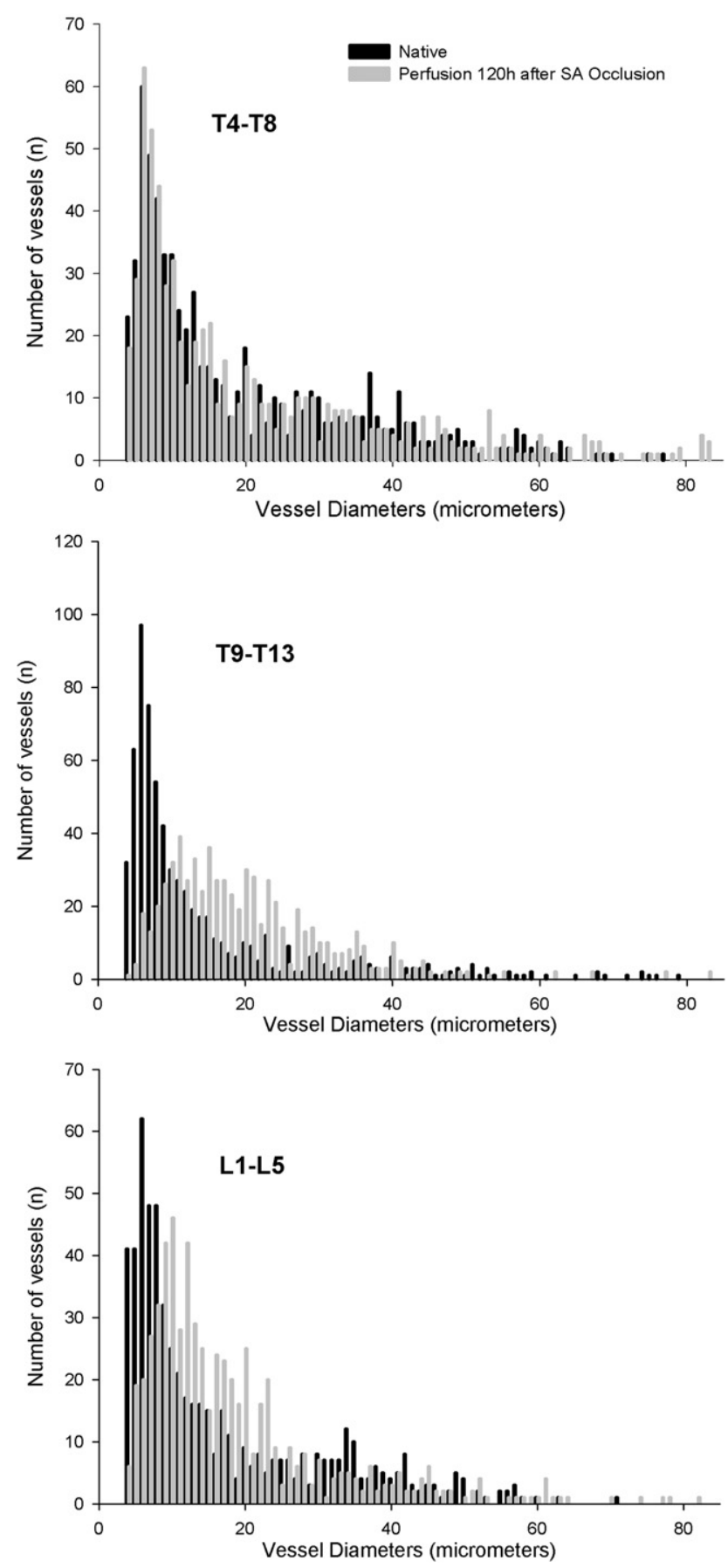

FIGURE 6. Shift in vessel diameter distribution in the paraspinous network 120 hours after complete SA ligation in the pig. In the lower thoracic and lumbar regions, a clear shift from 1 - to $10-\mu \mathrm{m}$ vessels (capillaries) to 10 to $30-\mu \mathrm{m}$ vessels (small arterioles) is apparent. $S A$, Segmental artery.

may be important in maintaining blood flow to the muscles themselves after SA sacrifice, but because there are connections at each segmental level between the intraspinal and paraspinous vessels, it is possible that flow can be directed both to the cord from the paraspinous muscles and away 

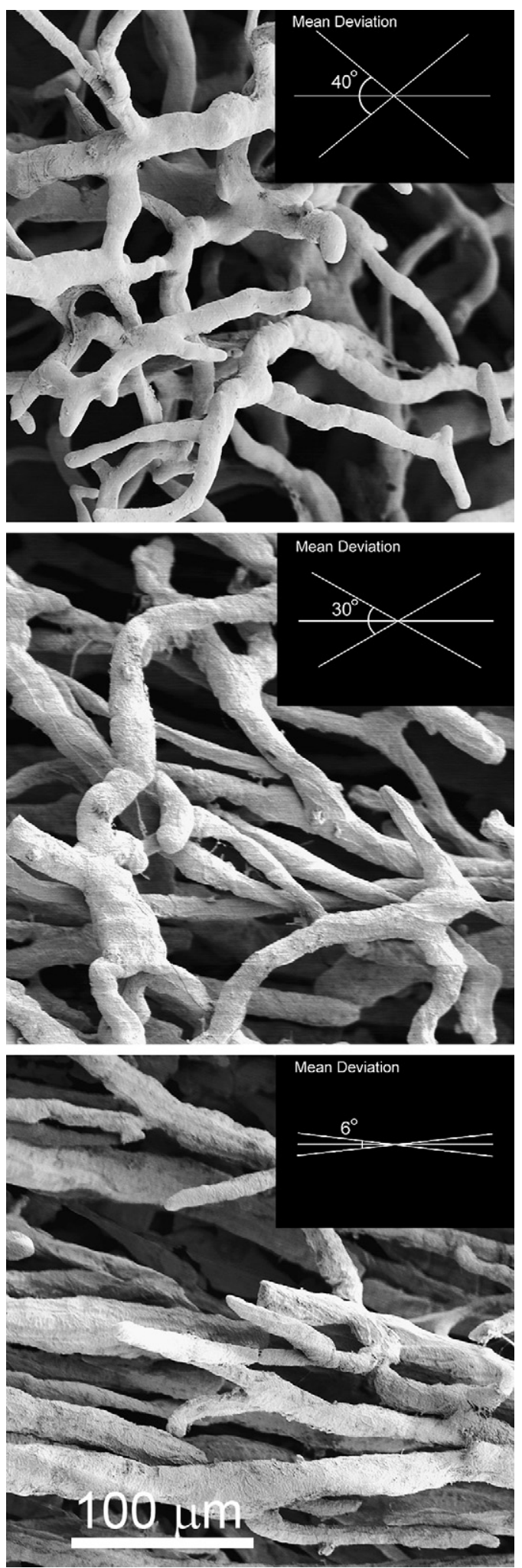

FIGURE 7. Progressive parallelization of small vessels in the paraspinal network 24 and 120 hours after SA ligation in the pig. Representative details from SEM images of the paraspinous vascular system show the change in vessel orientation after occlusion of segmental inflow into the spinal col- from it (steal), depending on the circumstances. In parallel with the changes in the paraspinous circulation, the ASA increases in diameter most markedly at its caudal and cranial extremes, helping to direct flow to the vulnerable midportion of the spinal cord.

As does the ASA, the epidural collaterals, which normally connect the SAs with each other longitudinally and transversely, also increase in diameter. Of note, however, there is no significant increase in epidural vessel diameters until 5 days after SA occlusion, whereas the first changes are already seen in the ASA after 24 hours. The increased capacity of epidural collaterals by 5 days may contribute to enhanced flow from the paraspinous intersegmental connections into the spinal canal, and ultimately into the ASA, coincident with the recovery of spinal cord perfusion pressure. This, however, can only be hypothesized, because the experimental study described is descriptive in nature and provides no direct evidence of the functional importance of the different components of the collateral network.

An interesting question is to what extent these findings, which describe the formal process of vascular remodeling within the spinal collateral network for the first time, mesh with different concepts of remodeling and development of new vessels described in various other animal models, principally of peripheral arterial occlusive disease and coronary artery disease. ${ }^{11,12}$ There are 2 major concepts of reactive vascular remodeling after interruption of a conductive artery. ${ }^{12}$ The process of angiogenesis describes the growth of distal capillary diffusion surface area as a reaction to local hypoxia. In contrast, the term " arteriogenesis" refers to reactive remodeling and enlargement of preexisting small vessels in response to altered flow, probably as the result of changes in local mechanical forces on the endothelial cells. ${ }^{13-15}$

The increase in the diameters of intraspinal vessels after interruption of SA inflow into the collateral networks is probably best explained by the concept of arteriogenesis. The preexisting arteriolar network of vessels surrounding the spinal cord is likely to experience a fundamental change in mechanical forces on its cells after its source of inflow is shifted to its cranial and caudal extremes (ie, after sacrifice of the SAs, which normally provide more direct input). Although the methods used for vessel analysis in this study cannot differentiate between functional vasodilation and true vascular wall remodeling and growth, hypoxia sufficient to trigger angiogenesis is unlikely to have occurred within the spinal cord in this model, in which many of the animals recover from SA sacrifice without functional or histologic evidence of ischemic damage. In contrast, dramatic

lateral network. Insets show the calculated deviation of measured vessel angles for the images. Top: native animal with patent segmental inflow. Middle: animal 24 hours after SA occlusion. Bottom: animal 5 days after SA occlusion. All images were taken from low thoracic and high lumbar segments. 


\section{Orientation of the Paraspinous Collateral Network Arterioles prior to and after complete SA sacrifice}

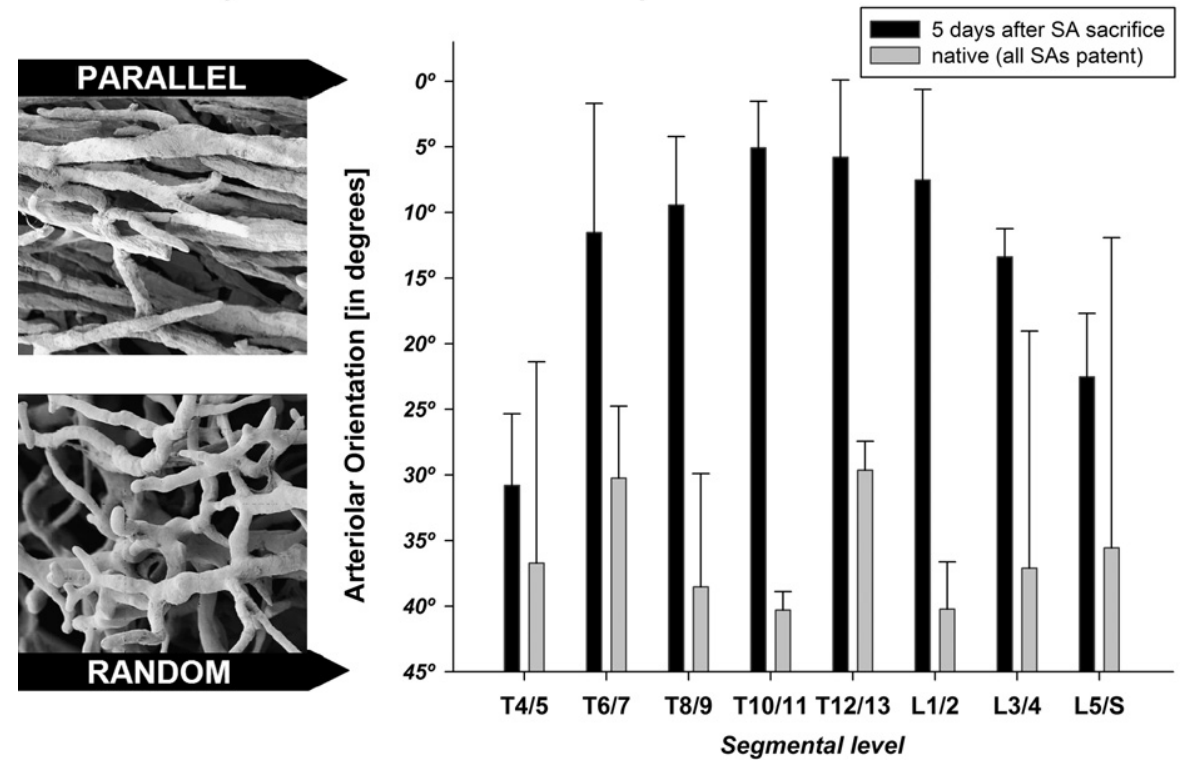

FIGURE 8. Extent of change in spatial orientation of paraspinous microvessels (arterioles up to $40 \mu \mathrm{m}$ ) 5 days after complete SA occlusion (T4-L5) compared with native pigs with patent segmental inflow into the collateral networks. For every segmental level, the mean orientation of all analyzed vessels was calculated. The graph shows the mean values of deviation from this calculated orientation: a random orientation of analyzed vessels results in higher values (45 degrees). Values closer to zero represent a vascular network with a unidirectional pattern. The images on the left show examples of both extremes: lower image, native vascular network; upper image, vascular network 5 days after SA occlusion. Note that the biggest differences in vascular orientation were found in the lower thoracic segments. $S A$, Segmental artery.

changes in flow dynamics are plausible after extensive SA sacrifice and have already been documented. ${ }^{4}$ If the mechanisms of the changes in the paraspinal vascular network can be explained using the principles of arteriogenesis, then pharmacologic and other means of improving the efficacy of this process, such as those used in peripheral vascular disease, ${ }^{16}$ also would be an option for enhancing the recovery of the spinal collateral network.

Although there is some justification for considering arteriogenesis to explain the observations within the intraspinal system after SA ligation, the findings after remodeling within the paraspinal muscles are more complex. The fact that the overall density of vessels within the paraspinous collateral network increases after SA sacrifice might suggest angiogenesis, but quantitative analysis reveals a capillary density decrease and an arteriolar capacity increase, which favors an arteriogenesis-like mechanism. The significance of the change in orientation of the paraspinous arterioles is not readily apparent, and has not been described in conjunction with angiogenesis or arteriogenesis. Further investigation is likely to illuminate these remaining questions.

Even without a more detailed understanding of the exact mechanisms by which the collateral circulation adapts, some clinical implications are already apparent. The vulnerability of the spinal cord to ischemia is clearly greatest in the first hours and days after SA sacrifice, when the changes involved in remodeling the collateral network are not yet fully developed or stabilized. ${ }^{2}$ The fact that the spinal cord perfusion pressure returns to baseline levels within 5 days is consistent with the current evidence of the rapid remodeling of the collateral network to augment flow from both cranial and caudal inputs. ${ }^{10}$ The idea that extreme vigilance in maintaining arterial pressures high and stable needs only to be sustained for a few days after extensive SA sacrifice to prevent spinal cord injury makes this effort a feasible proposition.

Although the triggers for the remodeling that takes place have not yet been defined, the timeline and character of the changes can be used already to help protect spinal cord integrity. For example, knowing that the collateral network is an integral part of the recovery of spinal cord blood supply makes it clear that preventing steal from the muscles adjacent to the cord - which are competing for a reduced supply of blood-is important. Thus, prolonging hypothermia to reduce metabolic requirements both within the cord and in the adjacent muscles, and inhibiting shivering during awakening from anesthesia could both potentially be helpful. Obviously, the vulnerability of the spinal cord to injury postoperatively is enhanced if there has already been intraoperative ischemia, so use of adjuncts_-including cerebrospinal fluid 
drainage-is important to minimize metabolic requirements and maintain adequate perfusion intraoperatively. Sensitive neuromonitoring will also allow detection and prompt correction of any intraoperative ischemia, should it occur.

Postoperatively, the first 24 hours are clearly a time of great vulnerability. Thus, SSEP monitoring of function and monitoring of the pressure in the collateral network are both potentially critical during this early postoperative interval to ensure that systemic arterial pressures are maintained at levels adequate to prevent ischemia. The ability to continue drainage of cerebrospinal fluid is also important for at least the first 24 hours postoperatively to facilitate spinal cord perfusion.

If the anatomic and physiologic response to reduction of SA input can be stabilized within 5 days, as suggested by the combination of the current findings and previous studies of spinal cord perfusion pressure and spinal cord blood flow, ${ }^{2,4}$ this paves the way for 2-stage strategies for repair of extensive TAAA. Other clinical and experimental studies have already shown that the rate of spinal cord injury is reduced with a 2-stage approach, and it can now readily be imagined that this is because the sacrifice of SAs during the first stage provokes definite anatomic adaptations to increase collateral blood supply. Such staged approaches could include hybrid procedures involving open surgery and endovascular techniques, as well as sequential endovascular approaches. A 2-stage approach might use temporary landing zones in treating extensive aneurysms that might not be suitable for long-term therapy, but would likely be safe for 1 or 2 weeks. The reduced risk of spinal cord ischemia associated with a 2-stage approach would allow more latitude in finding good permanent proximal and distal landing sites-including longer stretches of normal aorta-without fear of provoking paraplegia. The use of 2-stage procedures might also obviate the need for custom-designed branched grafts for use in extensive aneurysms.

Another possible approach may be to try to initiate the vascular remodeling seen in this study by coiling or embolizing some SAs as an initial procedure, before surgical or endovascular occlusion of the remaining SAs during aneurysm repair. Without knowing the stimulus for the remodeling of the collateral circulation, however, this approach would have to be explored first in an experimental model. At present, one cannot even be sure that the same robust remodeling of the surrounding vascular bed would be achieved with sudden simultaneous occlusion of all the SAs-during stentgrafting - as with the more gradual occlusion accompanied by motor evoked potential monitoring (and mild hypothermia) used in this study and during open surgical repair.
There is no guarantee that the pig model is in all ways an accurate reflection of what happens in humans, although thus far the parallel findings from pressure monitoring and functional outcomes have proven striking. It is certainly conceivable that a young, healthy pig may have more capacity for remodeling the collateral network of vessels supplying the spinal cord than a frail, elderly patient with vascular disease.

\section{CONCLUSIONS}

The current study holds out the promise that with proper attention to the pathophysiology of the spinal cord circulation, the threat of paraplegia can be reduced to levels not previously achievable. It is our hope that spinal cord injury will soon be a rare complication of repair of extensive TAAA.

\section{References}

1. Maniar HS, Sundt TM III, Prasad SM, Chu CM, Camillo CJ, Moon MR, et al. Delayed paraplegia after thoracic and thoracoabdominal aneurysm repair: a continuing risk. Ann Thorac Surg. 2003;75:113-20.

2. Etz CD, Luehr M, Kari FA, Bodian CA, Smego D, Plestis KA, et al. Paraplegia after extensive thoracic and thoracoabdominal aortic aneurysm repair: does critical spinal cord ischemia occur postoperatively? J Thorac Cardiovasc Surg. 2008; 135:324-30.

3. Etz CD, Halstead JC, Spielvogel D, Shahani R, Lazala R, Homann TM, et al. Thoracic and thoracoabdominal aneurysm repair: is reimplantation of spinal cord arteries a waste of time? Ann Thorac Surg. 2006;82:1670-7.

4. Etz CD, Di Luozzo G, Zoli S, Lazala R, Plestis KA, Bodian CA, et al. Direct spinal cord perfusion pressure monitoring in extensive distal aortic aneurysm repair. Ann Thorac Surg. 2009;87:1764-74.

5. Etz CD, Kari FA, Mueller CS, Silovitz D, Brenner RM, Lin HM, et al. The collateral network concept: a reassessment of the anatomy of spinal cord perfusion. J Thorac Cardiovasc Surg. 2010;8:1-9.

6. Verli FD, Rossi-Schneider TR, Schneider FL, Yurgel LS, de Souza MA. Vascular corrosion casting technique steps. Scanning. 2007;29:128-32.

7. Strauch JT, Lauten A, Zhang N, Wahlers T, Griepp RB. Anatomy of spinal cord blood supply in the pig. Ann Thorac Surg. 2007;83:2130-4.

8. Wei W, Popov V, Walocha JA, Wen J, Bello-Reuss E. Evidence of angiogenesis and microvascular regression in autosomal-dominant polycystic kidney disease kidneys: a corrosion cast study. Kidney Int. 2006;70:1261-8.

9. Etz CD, Homann TM, Luehr M, Kari FA, Weisz DJ, Kleinman G, et al. Spinal cord blood flow and ischemic injury after experimental sacrifice of thoracic and abdominal segmental arteries. Eur J Cardiothorac Surg. 2008;33:1030-8. Epub 2008 Apr 11.

10. Etz CD, Homann TM, Plestis KA, Zhang N, Luehr M, Weisz DJ, et al. Spinal cord perfusion after extensive segmental artery sacrifice: can paraplegia be prevented? Eur J Cardiothorac Surg. 2007;31:643-8.

11. Eitenmuller I, Volger O, Kluge A, Troidl K, Barancik M, Cai WJ, et al. The range of adaptation by collateral vessels after femoral artery occlusion. Circ Res. 2006; 99:656-62.

12. Heil M, Eitenmuller I, Schmitz-Rixen T, Schaper W. Arteriogenesis versus angiogenesis: similarities and differences. J Cell Mol Med. 2006;10:45-55.

13. Cai W, Schaper W. Mechanisms of arteriogenesis. Acta Biochim Biophys Sin (Shanghai). 2008;40:681-92.

14. Deindl E. Arteriogenesis: a focus on signal transduction cascades and transcription factors. Thromb Haemost. 2007;98:940-3.

15. Semenza GL. Vasculogenesis, angiogenesis, and arteriogenesis: mechanisms of blood vessel formation and remodeling. J Cell Biochem. 2007;102:840-7.

16. Schirmer SH, van Nooijen FC, Piek JJ, van Royen N. Stimulation of collateral artery growth: travelling further down the road to clinical application. Heart. 2009;95:191-7. 